\title{
VERIFICATION OF THE CROWN WALL STABILITY TAKING INTO ACCOUNT THE HYDRAULIC PERFORMANCE CURVES
}

\author{
Montse Vílchez ${ }^{1}$, Pilar Díaz-Carrasco ${ }^{1}$, María Clavero ${ }^{1}$, Miguel Ortega-Sánchez ${ }^{1}$, Miguel A. Losada ${ }^{1}$ \\ The design of a breakwater requires that the failure modes which can affect its security, functionality and operationality \\ in its useful life are bounded. The failure modes can be verified by means of the corresponding verification equation. \\ In this work, a unified methodology to verify simultaneously the failure modes produce by wind waves in front or \\ face of the breakwater is presented. This methodology is based on formulate the verification equations of the failure \\ modes forced by wind waves in the front or face of the breakwater in terms of the kinematic and dynamic variables \\ of the flow at these locations. It has been applied to analyse the failure mode that considers sliding of the crown wall. \\ Results show that the parameters of the pdf of these variables depend on the total wave height at the same location and \\ therefore, the verification equation can be also formulated in terms of this variable.
}

Keywords: Hydraulic performace; Sliding; Crown wall stability; Failure mode, Pressure, Forces

\section{INTRODUCTION}

A breakwater is a maritime structure built to protect a harbor, sheltered area, or shoreline from the full impact of waves. Breakwaters differ in the way that they deal with impinging waves. The selection of a breakwater type and the dimensioning of its sections and elements depend on the project requirements, site characteristics and wave climate. The most advanced technical criteria (ROM 1.0, 2009; WG47, 2013) establish that the project of the breakwater must address the requirement that the joint probability of failure for the principal modes of failure under ultimate and serviceability limit states must be bounded both during the service life and during different project stages. Currently, this verification is formulated independently for the different failure modes, even in the case in which the failure modes are affected by the same atmospheric and maritime agents. What is more, agents are considered without taken into account their transformation produced by the presence of the structure. Therefore, to optimize the breakwater design, the main failure modes affected by the wind waves should be verify simultaneously in a homogeneous way. This is only possible if the different verification equations are formulated including the total wave characteristics in the different locations in which the failure modes are taken place. Vílchez et al. (2016) applied a unified function to characterize the hydraulic performance of different breakwater typologies. This results permit to calculate the total wave height distribution in different locations in front and section of different breakwater typologies (Vílchez et al., 2017). Based on these results, the objective of this work is to analyze the crown wall stability as a function of the total wave height at the wall of the breakwater and to propose a new methodology to calculate the failure probability of the breakwater associated to the failure mode sliding of the crown wall.

\section{PROBLEM FORMULATION}

The verification equation for a failure mode consists in a set of terms which can be classified as favorable, $Z_{1}$, and unfavorable, $Z_{2}$. The safety margin is thus defined as the difference between both terms: $S=Z_{1}-Z_{2}$. The sliding of the superestructure failure mode occurs when the resulting wave force in the seaward direction exceeds the friction force between the caisson base and the bedding layer. The terms of the verification equation for this failure mode and a breakwater with geometry as shown figure 1 are the following: 1) Favorable term: $Z_{1}=\mu_{c}\left(W_{1}-F v\right)$;2) Unfavorable term: $Z_{2}=F h . \mu_{c}$ is the friction coefficient between the caisson base and the bedding layer, $W_{1}$ is the submerged weight of the caisson (equation 1) and $\gamma_{c}$ and $\gamma_{w}$ are the specific weight of the caisson and water respectively. Horizontal and vertical forces in the wave crest ( $F h$ and $F v$ ) can be obtained by integrating the pressure laws (equation 2 and 3).

$$
\begin{gathered}
W_{1}=\left[F_{c}+\left(h-h_{b}\right)\right] B \gamma_{c}-\left[\left(h-h_{b}\right) B \gamma_{w}\right] \\
F h=\int_{-\left(h-h_{b}\right)}^{\eta} P\left(x=x_{w}\right) d z \\
F v=\int_{x_{w}}^{x_{w}+B} P\left(z=-\left(h-h_{b}\right)\right) d x
\end{gathered}
$$

\footnotetext{
${ }^{1}$ Environmental Fluid Dynamics Group. University of Granada. Spain.
} 
$P$ is the pressure in the wave crest, $\eta$ is the water elevation in the wave crest, $z$ is the vertical coordinate, $x$ is the horizontal coordinate, $x_{w}$ is the wall location.

\section{Dynamic pressure at the breakwater wall}

The instantaneous free surface elevation, $\eta_{t}(x, t)$ (results from the linear superposition of the incident and reflected wave trains), at any location in front of the coastal structure has a Gaussian probability density function (Vílchez et al., 2017). Dynamic pressure $(p)$ is shown to be statistically independent and to possess a Gaussian probability distribution (Borgman, 1967). $p$ at the breakwater wall can be calculated, in a complex form, in terms of the total free surface elevation by means of the transfer function, $F_{t p}$ :

$$
p\left(t, x=x_{w}, z\right)=F_{t p}(f, z) \eta_{t}\left(t, x=x_{w}\right)
$$

where

$$
F_{t p}(f, z)= \begin{cases}\rho g \frac{\cosh (k(h+z))}{\cosh (k h)} & \text { if }-\left(h-h_{b}\right)<z \leq 0 \\ \rho g & \text { if } 0 \leq z<\eta_{t}\end{cases}
$$

$\rho$ is the water density, $g$ is the gravity acceleration and $k$ is the wave number. The corresponding pressure spectra is given as:

$$
S_{p}\left(f, x=x_{w}, z\right)=\left|F_{t p}(f, z)\right|^{2} S_{t}\left(f, x=x_{w}\right)
$$

When the incident waves have a narrow-band spectrum, the transfer functions can be approximated to a function of a representative central frequency, $f_{0}$, (Vílchez et al., 2017). Defining $P$ as the pressure in the wave crest (for each wave of the sea state), the root mean square value of $P$, can be then calculated as a function of the r.m.s wave height at the breakwater wall, $H_{w r m s}$, as follows:

$$
P_{r m s}\left(x=x_{w}, z\right)= \begin{cases}\rho g \frac{\cosh \left(k_{0}(h+z)\right)}{\cosh \left(k_{0} h\right)} \frac{H_{w r m s}}{2} & \text { if }-\left(h-h_{b}\right)<z \leq 0 \\ \rho g\left(\frac{H_{w r m s}}{2}-z\right) & \text { if } 0 \leq z<\eta_{t}\end{cases}
$$

\section{METHODOLOGY}

Three different breakwater typologies have been analyzed (figure 1): Composite breakwater (Type B); Composite breakwater protected by a porous submerged berm (Type C), and rubble mound breakwater with crown wall (Type D). For Type B, experimental data of composite breakwater by Clavero et al. (2012) were reanalyzed. The model consisted of a composite breakwater with rectangular cross section. Tests were done in the wave flume of CEAMA -University of Granada $(23 \times 0.65 \times 1 \mathrm{~m})$. Three different model widths $(B=0.14,0.50$ and $1.50 \mathrm{~m})$, two different relative foundation depth $\left(h_{b} / h=0.25\right.$ and 0.50$)$ and five different granular nominal diameter $(D=12,26,40,52$ and 110) were tested. Wave period was varied from $T_{p \text { teo }}=1-3$ seconds with increment of 0.25 seconds and for each period the wave height was varied from $H_{\text {Is teo }}=0.04-0.10 \mathrm{~m}$ with increments of $0.02 \mathrm{~m}$. Water depth was kept constant and equal to $0.4 \mathrm{~m}$. Test were done with non- overtopping and non-breaking conditions. Eight resistance wave gauges (figure 2) and eight pressure sensors were installed and used to measure free surface elevations and pressure respectively, with a sampling frequency of $20 \mathrm{~Hz}$.

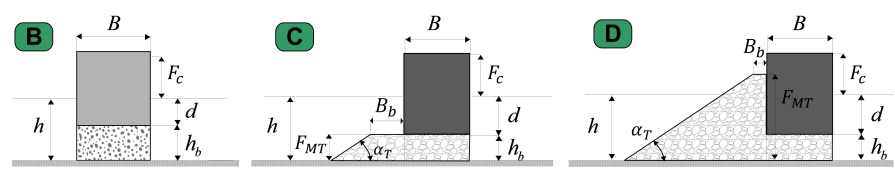

Figure 1: Breakwater geometries.

A set of numerical simulations have been done with the IH-2VOF model (Lara et al., 2008) to test Type $\mathrm{C}$ and D. The breakwater tested consisted of a composite breakwater protected by a porous berm. Three different relative berm height $\left(F_{M T} / h=0.50,0.75,1.00\right)$ for Type $\mathrm{C}$ and two $\left(F_{M T} / h=1.25,1.50\right)$ for Type $\mathrm{D}$ were tested. For both types, two different relative berm heights $\left(B_{b} / h=0.250\right.$ and 0.625$)$ have been tested and $\mathrm{D}=40 \mathrm{~mm}$. The slope angle was $1: 1.5$ and water depth was $\mathrm{h}=0.40 \mathrm{~m}$. Different wave periods $\left(T_{p \text { teo }}\right.$ 


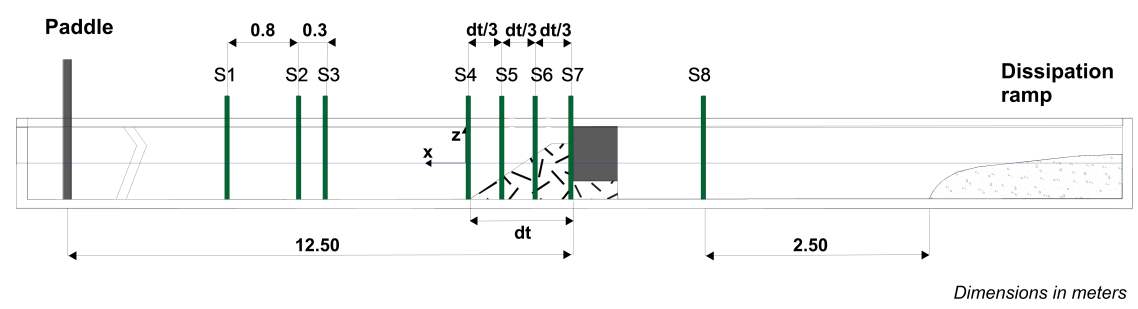

Figure 2: Description of experimental set-up.

$=1.05,1.50,2.00$ and $2.50 \mathrm{~s})$ and wave heights $\left(H_{\text {Is teo }}=0.04,0.06 \mathrm{~m}\right)$ were tested for irregular waves and non-overtoppable conditions. The CEAMA -University of Granada wave flume was reproduced in the IH2 VOF model using a 2DV domain. A mesh sensitivity analysis was performed to assess the computational cost and the accuracy of the results. The total number of cells in the numerical domain was $1304 \times 162$. Active wave absorption was considered at the generation boundary, and the dissipative ramp at the final of the flume was reproduced with a porous medium. The friction in the porous medium was calibrated following (Vílchez et al., 2016b). The data obtained from the numerical model was the same that from the experimental tests. For each test or simulation, the following variables were obtained (figure 3):

- Experimental reflection coefficient modulus $\left(K_{R}\right)$ and phase $(\phi)$ were calculated by using two set of three sensors (S1,S2,S3) and applying the method proposed by Baquerizo (1995). Transmission coefficient $\left(K_{T}\right)$ with sensor S8.

- The total free surface elevation at the breakwater wall $\eta_{w}(t)$ was measured using the sensor S7. $H_{w r m s}$ : Root mean square value of the free surface elevation at the wall.

- $p(t), F H(t)$ and $F V(t)$ : Time series of pressure in different locations along the wall and horizontal and vertical forces.

- Horizontal pressure distribution: 1) $P_{1 r m s}$ : Root mean square value of the pressure at the mean water level; 2) $P_{2 r m s}$ : Root mean square value of the pressure at the bottom of the caisson. Vertical pressure distribution: 1) $P_{u 1 r m s}$ : Root mean square value of the pressure at the entrance of the porous medium; 2) $P_{u 2 r m s}$ : Root mean square value of the pressure at the end of the porous medium.

- $F h$ and $F v$ : Horizontal and vertical force in the wave crest for each wave of a sea state and the root mean square value of these variables $\left(F h_{r m s}\right.$ and $F v_{r m s}$ respectively).

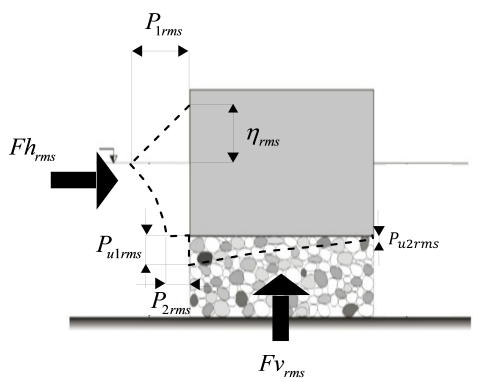

Figure 3: Scheme of pressure laws and analyzed variables.

\section{RESULTS}

Horizontal pressure distribution

Pressure over the mean water level

It is known (Goda, 1985; Takahashi, 1996) that the pressure law above S.W.L. follows a triangular distribution depending on the maximum sea water level elevation at the wall. Figures 4 and 5 show the 
r.m.s value of the pressure at the mean water level, $P_{1 r m s}$, compared with the hydrostatic pressure at this location calculated as given by equation 7 for $\mathrm{z}=0$. These results are presented for type $\mathrm{B}$ as a function of $h_{b} / h$ (figure 4) and for type $\mathrm{C}$ and D as a function of $F_{M T} / h$ and $B_{b} / h$ (figure 5).
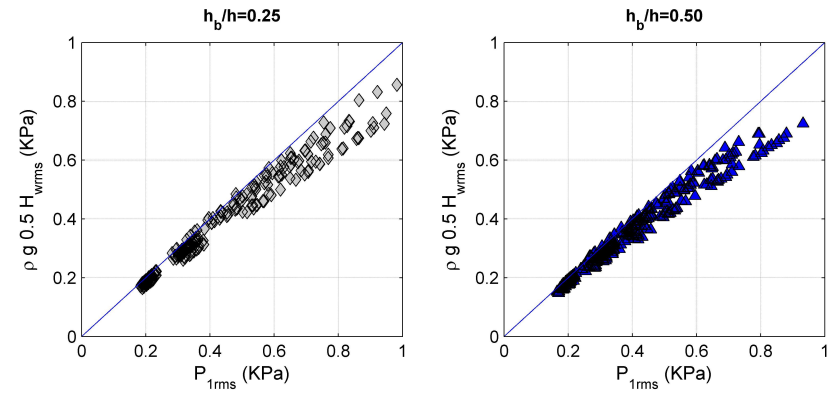

Figure 4: Comparison between numerical and theoretical value of the pressure at the S.W.L. for type B as a function of $h_{b} / h$.
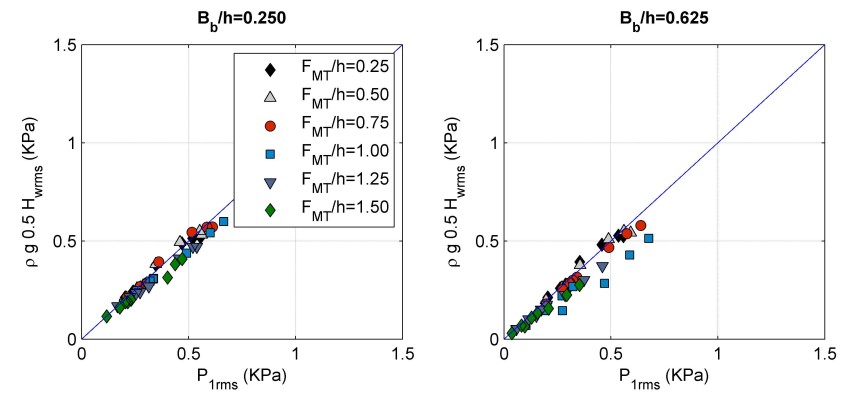

Figure 5: Comparison between numerical and theoretical value of the pressure at the S.W.L. for type C and $D$ as a function of $F_{M T} / h$ and $B_{b} / h$.

For all typologies, it is verified that the pressure over the mean water level is directly proportional to the sea water elevation at the wall. For larger pressures, the theoretical expression underestimates the pressure at this location. This can be because of the nonlinear effects produced in the wave train when the wave reaches the wall. To correct this effect, a coefficient, $\alpha_{1}$, has been included (equation 8). $\alpha_{1}=1.14$ has been found for all breakwater typologies. Moreover, for HMCB, $F_{M T} / h=1.00$ and the largest berm, $B_{b} / h=0.625$, pressures greater than the ones obtained with equation 8 were observed. This overpressure can be due to impulsive pressures that were observed at the wall for the smallest wave period. In this case, the value of $P_{1 \mathrm{rms}}$ can be calculated as the modified hydrostatic pressure affected by an empirical coefficient, $\alpha_{I}$, to take into account this excess of pressure (equation 9).

$$
\begin{gathered}
P_{1 r m s}=\rho g \frac{H_{w r m s}}{2} \alpha_{1} \\
P_{1 r m s}=\left[\rho g \frac{H_{w r m s}}{2} \alpha_{1}\right] \alpha_{I}
\end{gathered}
$$

$\alpha_{I}$ depends mainly on the parameter $B_{b} / h$ and $H_{\text {Irms }} / h$ (figure 6). The excess of pressure increases with the relative berm width and relative wave height increase. This result is in accordance with Takahashi (1996), but this author proposed higher values of $\alpha_{I}$ because the analysis was done without taking into account the reflection processes in the total wave height calculation. The best fits to calculate $\alpha_{I}$ for HMCB typology are also included in figure 6. For the rest of cases $\alpha_{I}=1$.

Pressure below the mean water level

Figure 7 shows the relationship between the root mean square of the non-dimensional values of the pressure at the mean water level, $P_{1 r m s}$, and at the bottom of the caisson, $P_{2 r m s}$, for type B. For the two 


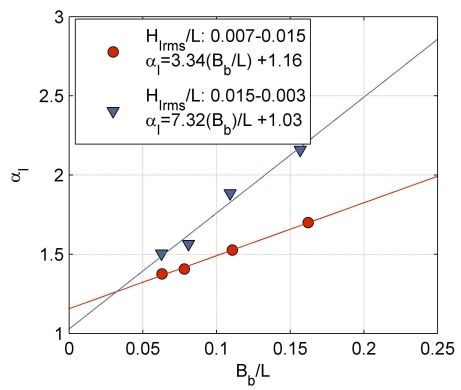

Figure 6: Impulsive pressure coefficient, $\alpha_{I}$, for typology HMCB as a function of $B_{b} / L$ and $H_{I r m s} / h$.

analysed relative foundation depths, $h_{b} / h=0.25$ and $0.50, P_{2 r m s}$ mainly depends on the relative depth, $k h$. Long waves produce the greater pressure at the bottom. $P_{2 r m s}$ has been compared with the theoretical pressure at this location, $z=-\left(h-h_{b}\right)$, (equation 7).

$$
P_{2 r m s}=P_{1 r m s} \frac{\cosh \left(k_{0} h_{b}\right)}{\cosh k_{0} h}
$$

In this work, $f_{0}$ has been consider as the peak frequency. Non-dimensional results are shown in figure 8 for type B with two relative foundation depths. Equation 10 correctly represents the results but slightly overestimates the value of $P_{2 r m s}$ for the largest pressures and the two relative foundation depths. This head loss could be due to the sudden increase of velocity at the entrance of the porous medium. Consequently, the pressure decreases in this location.
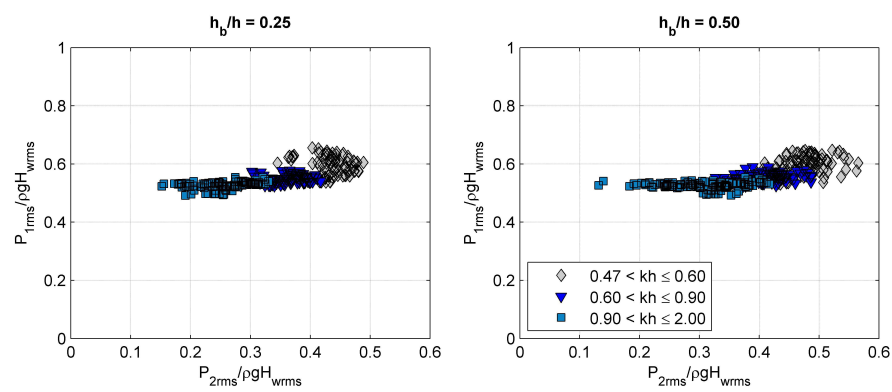

Figure 7: Relationship between non-dimensional pressure at the S.W.L., $P_{1 \mathrm{rms}}$, and the pressure at the bottom of the caisson, $P_{2 r m s}$, for type $\mathbf{B}$ as a function of $h_{b} / h$ and $k h$.

The same results are found for type C and D. An example of comparison between numerical and theoretical pressure laws below S.W.L. is shown in figure 9 for $T_{p \text { teo }}=2.5 \mathrm{~s}$ and $H_{I s \text { teo }}=0.08 \mathrm{~m}$. The blue line represents the location of the breakwater berm coronation. For LMB, $F_{M T} / h=0.25$ and 0.50 , numerical pressure law below S.W.L. sets very successfully with theoretical law. In this case, the pressure reduction effect is not so pronounced than the one observed for type B. The presence of the berm could damp the sudden change in the stream line at the entrance of the porous medium. For $F_{M T} / h \geq 0.75$ the berm or slope is total or partially protecting the crown and the presence of the granular material attenuates the value of $P_{2 r m s}$. For HMB, $F_{M T} / h=0.75$, the pressure law is well fitted to the hyperbolic cosine law (equation 10) until the point in which there is a change of medium (only water $\rightarrow$ water + granular material) at $z /\left(h-h_{b}\right)=$ -0.5 . From this location the pressure is slightly lower than the one given by the theoretical law. The biggest difference is observed for HMCB, $F_{M T} / h=1.00$. Finally, for RMB-CW, $F_{M T} / h>1.00$, the complete pressure law from the S.W.L. to the bottom of the caisson is affected by the presence of the porous medium and the pressure deficit is reduced in comparison with the two previous typologies. In order to quantify this pressure deficit, a new coefficient $\alpha_{2}$ has been introduced (Equation 11), $\alpha_{2}=f\left(h_{b} / h, F_{M T} / h\right)$. Values of $\alpha_{2}$ for the different typologies are presented in the table 1 . The pressure law could be approximated by a 
linear distribution from the S.W.L. and the bottom of the caisson for all breakwater typologies, with $P_{2 r m s}$ calculated as given in equation 11 . In the case of $F_{M T} / h=0.75$, the horizontal law would be composed by two different distributions: 1) a linear law between the S.W.L. and the berm coronation, in which pressure, $P_{12 r m s}$, can be calculated with equation 11 taken $\alpha_{2}=1$ and 2) other linear law from this point to the bottom of the caisson where $P_{2 r m s}$ can be calculated with equation 11 taken $\alpha_{2}$ as given in table 1 .
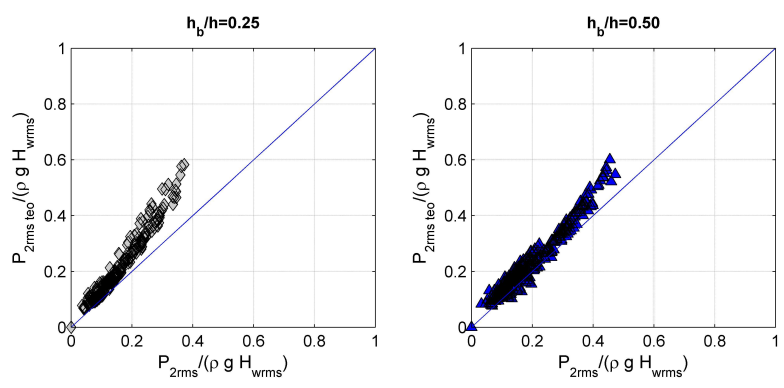

Figure 8: Comparison between non-dimensional pressure at the bottom of the caisson, experimental, $P_{2 r m s}$, and theoretical, $P_{2 r m s t e o}$, for type $\mathbf{B}$ as a function of $h_{b} / h$.

$$
P_{2 r m s}=P_{1 r m s} \frac{\cosh \left(k_{0} h_{b}\right)}{\cosh k_{0} h} \alpha_{2}
$$
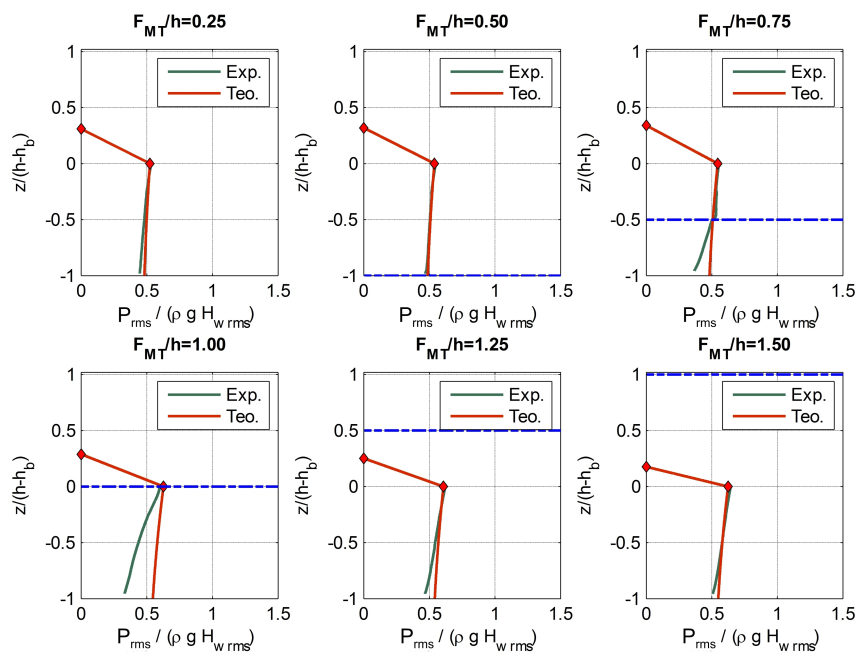

Figure 9: Comparison between horizontal pressure laws for type $\mathbf{C}$ and $\mathbf{D}$ as a function of $F_{M T} / h$. $T_{p \text { teo }}=\mathbf{2 . 5 0} \mathrm{s}, H_{\text {Is teo }}=\mathbf{0 . 0 8} \mathbf{m}$ and $B_{b} / h=\mathbf{0 . 2 5 0}$.

\section{Uplift pressure distribution}

The uplift pressures will depend mainly on the wave transformation processes in the porous medium. Firstly, the shape of the uplift pressure law is analysed. Figure 10 show the non-dimensional uplift pressure along the bottom of the caisson for type $\mathrm{C}$ and $\mathrm{D}$. Results are presented as a function of the coordinate $x^{\prime} / B$ ( $x^{\prime}$ is the local coordinate, with $x^{\prime}=0$ at the entrance of the porous medium) in terms of $F_{M T} / h$ and $k h$ for $B_{b} / h=0.250$. The uplift pressure law is nearly linear which indicates that the flow in the porous medium is fully developed (Losada et al., 1993). Long waves increase the uplift pressure at the entrance of the porous medium. Moreover, in the most of the cases uplift pressure at the exit is not zero. The behavior is the same for the two values of $B_{b} / h$.

The value of the non-dimensional uplift pressure at the entrance of the porous medium, $P_{u 1 r m s}$, is compared with the non-dimensional pressure at the bottom of the caisson, $P_{2 r m s}$ for type B (figure 11) 


\begin{tabular}{ccc}
\hline Typology & $h_{b} / h$ or $F_{M T} / h$ & $\alpha_{2}$ \\
\hline \hline \multirow{2}{*}{ CB } & $h_{b} / h=0.25$ & 1.38 \\
& $h_{b} / h=0.50$ & 1.17 \\
\hline \multirow{2}{*}{ LMB } & $F_{M T} / h=0.25$ & 1 \\
& $F_{M T} / h=0.50$ & 1 \\
\hline HMB & $F_{M T} / h=0.75$ & 0.31 \\
\hline HMCB & $F_{M T} / h=1.00$ & 1.70 \\
\hline \multirow{2}{*}{ RMB-CW } & $F_{M T} / h=1.25$ & 1.17 \\
& $F_{M T} / h=1.50$ & 1.09 \\
\hline
\end{tabular}

Table 1: $\alpha_{2}$ values for the different typologies.
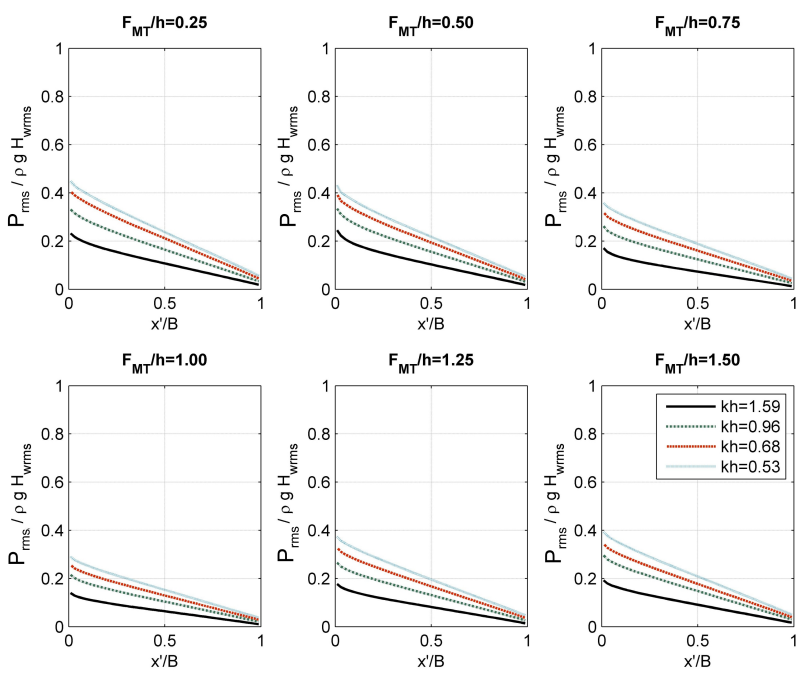

Figure 10: Uplift pressure laws for type $\mathbf{C}$ and $\mathrm{D}$ as a function of $F_{M T} / h$ and $k h . B_{b} / h=\mathbf{0 . 2 5 0}$.

and $\mathrm{C}$ and D (figure 12). For type B, results are shown for the two relative foundation depths, $h_{b} / h$, as a function of the relative width of the caisson, $B / L$ and $D k$. In general, there is a linear relationship between the pressure values, although $P_{u 1 r m s}$ is slightly lower than $P_{2 r m s}$ because of the change in the stream line direction at the entrance of the porous medium. The head loss (value of $P_{u 1 r m s}$ relative to $P_{2 r m s}$ ) increases when $B / L$ decreases and for the highest $h_{b} / h$. The influence of $D k$ is only significant when $B / L<0.10$. Results are similar in the case of type $C$ and $\mathrm{D}$ where $B / L$ was tested in the range of $0.10 \leq B / L<0.35$. For $B_{b} / h=0.250$ the head loss is negligible in all cases whereas for $B_{b} / h=0.625$ slightly increases for $F_{M T} / h \geq 1$. For the prediction of the uplift forces $P_{u 1 \mathrm{rms}}=P_{2 \mathrm{rms}}$ can be assumed.

Finally, the uplift pressure at the exit of the porous medium is analysed. At previously stated, the pressure at this location cannot be considered zero. It should depend on the transmission coefficient through the porous medium. Therefore, the relation between $P_{u 1 r m s}$ and $P_{u 2 r m s}$ has been studied as a function of $K_{T}$ as follows:

$$
P_{u 2 r m s} / P_{u 1 r m s}=\alpha_{3} K_{T}
$$

with $\alpha_{3}$ a empirical coefficient. It is presented in figure 13 for type $\mathrm{C}$ and $\mathrm{D}$, as example. This parameter depends mainly on the relative width of the caisson $B / L$. It decreases exponentially with $B / L$ until a value $B / L=(B / L)_{\min }$ from which $\alpha_{3}$ is nearly zero. Therefore, three types of uplift pressure laws can be defined as a function of $B / L$ :

- Trapezoidal uplift pressure law for $B / L<(B / L)_{\min }$. In this case the wave is not completely dissipated by the porous medium and pass leeward the structure.

- Triangular uplift pressure law for $B / L=(B / L)_{\min }$. In this case $P_{u 2 r m s}=0$. 

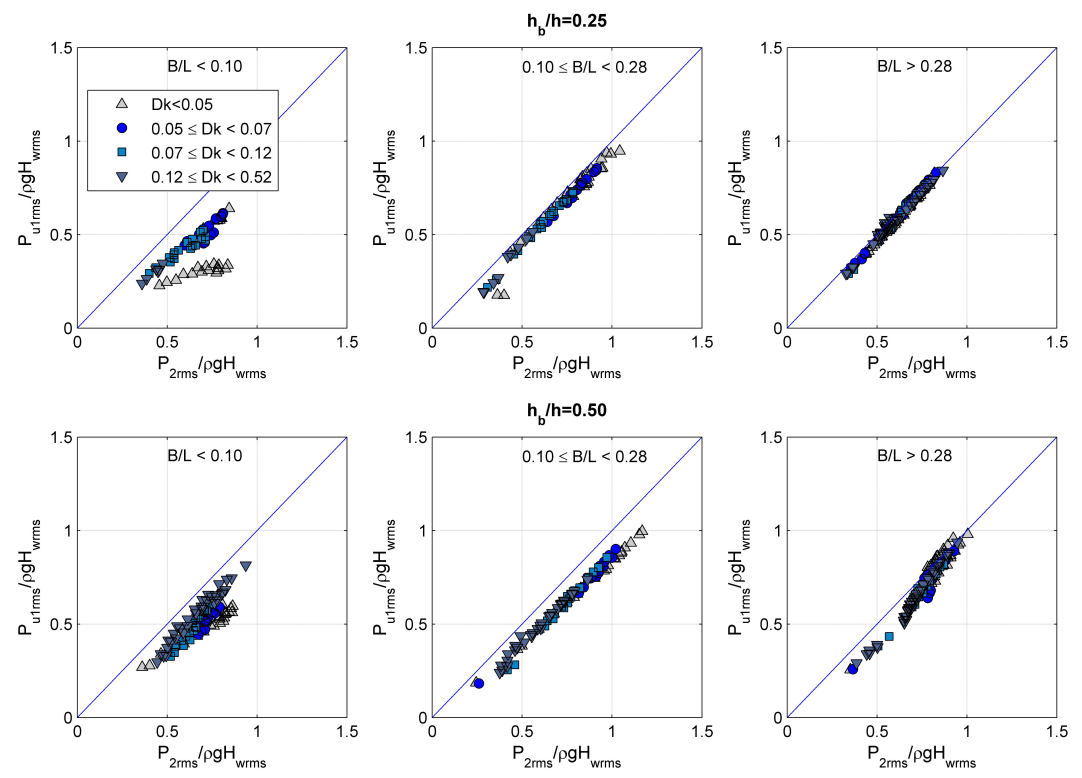

Figure 11: Relationship between non-dimensional pressure at the bottom of the caisson, $P_{2 r m s}$, and pressure at the entrance of the porous medium, $P_{u 1 r m s}$, for type $\mathbf{B}$ as a function of $h_{b} / h, D k$, and $B / L$.
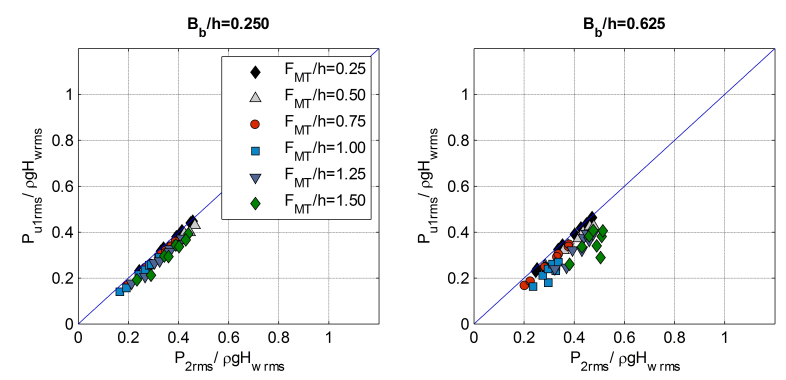

Figure 12: Relationship between non-dimensional pressure at the bottom of the caisson, $P_{2 r m s}$, and pressure at the entrance of the porous medium, $P_{u 1 r m s}$, for type $\mathbf{C}$ and $\mathbf{D}$ as a function of $F_{M T} / h$ and $B_{b} / h$.

- Triangular uplift pressure law with zero pressure before the exit of the caisson for $B / L>(B / L)_{\min }$. In this case the wave dissipates completely in the porous medium and there is not any wave transmission leeward the breakwater.

The $\alpha_{3}$ coefficient also depends on the breakwater typology $\left(D k, h_{b} / h, F_{M T} / h\right.$ and $\left.B_{b} / h\right)$ although to a lesser extent. Table 2 presents the equation to obtain $\alpha_{3}$ and $(B / L)_{\min }$ for types $\mathrm{B}, \mathrm{C}$ and $\mathrm{D}$. Moreover, the best fit curves for type $\mathrm{C}$ and $\mathrm{D}$ are presented in figure 13.

\section{Horizontal and vertical forces}

Figure 14 show the relationship between the non-dimensional r.m.s. values of the horizontal and vertical forces for type B. Data are separated for the two relative foundation depths and have been plotted as a function of the relative width of the caisson. Results show that data can be grouped for different $B / L$ intervals presenting a linear behavior. In all cases, the slope of the line increases with $B / L$. Three force regimes can be distinguished as a function of this parameter for the two relative foundation depths: 1) Regime in which the horizontal forces are dominants. This corresponds with the lowest range of $B / L$ $(B / L<0.10) ; 2)$ Regime in which the vertical forces are dominant corresponding with the highest range of $B / L(0.28<B / L<1.20) ; 3)$ Intermediate regime in which both forces have the same importance. This corresponds with the intermediate range of $B / L(0.10<B / L<0.28)$. 

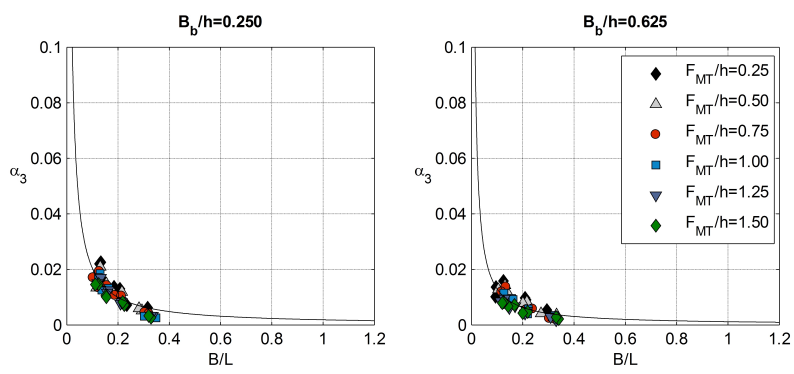

Figure 13: $\alpha_{3}$ as a function of $B / L, F_{M T} / h$ and $B_{b} / h$. Type $\mathbf{C}$ and $\mathbf{D}$.

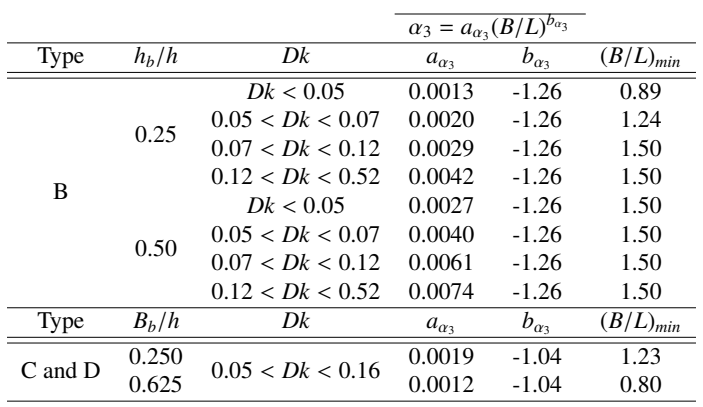

Table 2: $\alpha_{3}$ values for the different typologies.

Moreover, vertical forces increase with an increment of the foundation depth. For type C and D a linear relationship is also observed with the slope decreasing with an increase of $F_{M T} / h$. The dependence on this parameter is greater as the relative berm height increases. In these typologies the interval of $B / L$ was $0.1<B / L<0.35$, which corresponds with the intermediate regime and both forces are important. The slope of the line has been obtained. This value is represent by $\alpha_{4}$ (equation 13) which is included in table 3 for all typologies. This coefficient is a function of the breakwater typology.

$$
F v_{r m s}=\alpha_{4} F h_{r m s}
$$
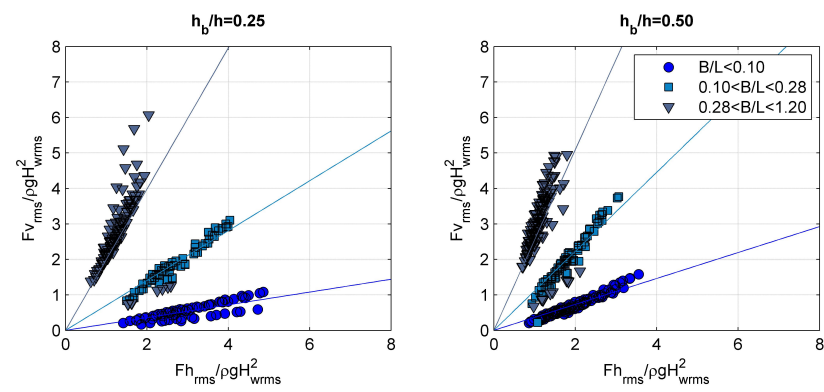

Figure 14: Relationship between r.m.s value of the non-dimensional horizontal and vertical forces as a function of $h_{b} / h$ and $D k$. Type B.

\section{Probability density function of the horizontal and vertical forces}

In existing formulae in literature, it is assumed that the maximum horizontal and vertical forces in a sea state occur simultaneously. However, several authors have pointed out the invalidity of said hypothesis (Vílchez et al., 2011). This has also been proved in this research. Figure 15 shows the maximum horizontal force and its respective vertical force in the crest $\left[F h_{\max }, F v_{x}\right]$ as well as the maximum vertical force and its respective horizontal force in the crest $\left[F h_{x}, F v_{\max }\right]$ for every wave of a sea state tested for the HMB typology. It can be observed that the maximum horizontal and vertical forces are not in phase. 


\begin{tabular}{|c|c|c|c|c|}
\hline Type & $h_{b} / h$ & $B / L$ & & $\alpha_{4}$ \\
\hline \multirow{6}{*}{ B } & \multirow{3}{*}{0.25} & \multicolumn{2}{|l|}{$B / L<0.10$} & 0.18 \\
\hline & & \multirow{2}{*}{\multicolumn{2}{|c|}{$\begin{array}{l}0.10 \leq B / L<0.28 \\
0.28 \leq B / L<1.20\end{array}$}} & 0.70 \\
\hline & & & & 1.99 \\
\hline & \multirow{3}{*}{0.50} & \multicolumn{2}{|l|}{$B / L<0.10$} & 0.37 \\
\hline & & \multirow{2}{*}{\multicolumn{2}{|c|}{$\begin{array}{l}0.10 \leq B / L<0.28 \\
0.28 \leq B / L<1.20\end{array}$}} & 1.11 \\
\hline & & & & 2.54 \\
\hline Type & $B_{b} / h$ & $B / L$ & $F_{M T} / h$ & $\alpha_{4}$ \\
\hline \multirow{12}{*}{ C and D } & \multirow{6}{*}{0.250} & \multirow{6}{*}{$0.10<B / L<0.35$} & 0.25 & 0.97 \\
\hline & & & 0.50 & 0.89 \\
\hline & & & 0.75 & 0.74 \\
\hline & & & 1.00 & 0.68 \\
\hline & & & 1.25 & 0.80 \\
\hline & & & 1.50 & 0.85 \\
\hline & \multirow{6}{*}{0.625} & \multirow{6}{*}{$0.10<B / L<0.35$} & 0.25 & 0.99 \\
\hline & & & 0.50 & 0.87 \\
\hline & & & 0.75 & 0.64 \\
\hline & & & 1.00 & 0.64 \\
\hline & & & 1.25 & 0.91 \\
\hline & & & 1.50 & 0.97 \\
\hline
\end{tabular}

Table 3: $\alpha_{4}$ values for the different typologies.

The maximum horizontal force is associated with a given vertical force, and the maximum vertical force is associated with a given horizontal force. Moreover, there is a combination of forces whose effect on the structure could be equal or even more unfavorable than the two combinations of maximum and their concomitant forces. Consequently, in order to design the crown of the breakwater, different combinations of horizontal and vertical forces should be analysed.

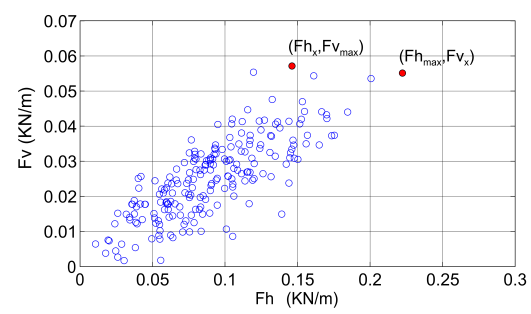

Figure 15: Simultaneous vertical and horizontal forces for HMB typology.

In this subsection the probability density functions of the horizontal and vertical forces in the wave crest are studied. Then, the joint probability density function of these variables has been obtained.

The probability density functions of the horizontal and vertical forces in the wave crest $(F h$ and $F v$ respectively) have been calculated for each sea state. Based on these results, the theoretical pdf is then obtained. Figure 16 display the empirical histogram of the non-dimensional value of $F h$ (left) and $F v$ (right) juxtaposed with the calculated histogram for type B with $B=0.14 \mathrm{~m}, h_{b} / h=0.25, D=40 \mathrm{~mm}, T_{p \text { teo }}=2.25 \mathrm{~s}$ and $H_{I s ~ t e o}=0.06 \mathrm{~m}$. The best fit for both variables, $F h$ and $F v$, has been found to be the Weibull probability density function.

The Weibull parameters, $\beta_{w}$ and $\delta_{w}$ for $F h$ y $F v$ are shown in figure 17 and 18 for type B and $F h$, as example. The scale parameter $\delta_{w}$ normalized with respect to the r.m.s value of the total wave height at the wall has been compared with the non-dimensional r.m.s of $F h$ or $F v$. For all three breakwater typologies, these two variables have a linear relation with 1:1 slope. Therefore this parameter could be accurately approximated to the root mean square value of the variable. The shape parameter, $\beta_{w}$, has been related to the shape parameter of the total wave height at the breakwater wall, $\beta_{w}\left(H_{w}\right)$, as a function of $k_{0} h$ (with $k_{0}$ the wave number at the breakwater toe), $h_{b} / h$ and $D k$ for type B. This relationship is approximately constant or increases slightly with $k_{0} h$ for both variables, $F h$ and $F v$, and for both relative foundation depths. However, for $F v$ data scattered increases. For type $\mathrm{C}$ and $\mathrm{D}$ two different behaviours are observed depending on the portion of the caisson that the berm is protecting. This parameter is constant or increases slightly with $k_{0} h$ for $F_{M T} / h \leq 1.00$ and decreases for $F_{M T} / h>1.00$. A weakly influence of $B_{b} / h$ has been observed. The 

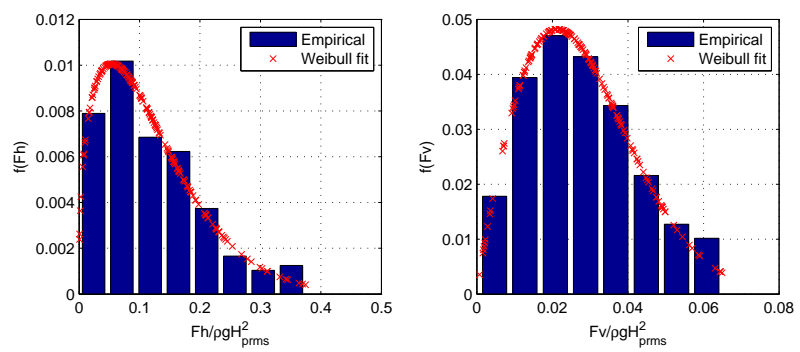

Figure 16: Probability density function of the non-dimensional horizontal (left figure) and vertical (right figure) force in the wave crest. Type $\mathbf{B}, B=0.14 \mathrm{~m}, h_{b} / h=0.25, D=40 \mathrm{~mm}, T_{p \text { teo }}=2.25 \mathrm{~s}$ and $H_{I s \text { teo }}=\mathbf{0 . 0 6} \mathbf{~ m}$. Empirical and theoretical fit (Weibull distribution).

best fit for the relationship between the shape parameters of the pdf of these two variables is given in table 4.
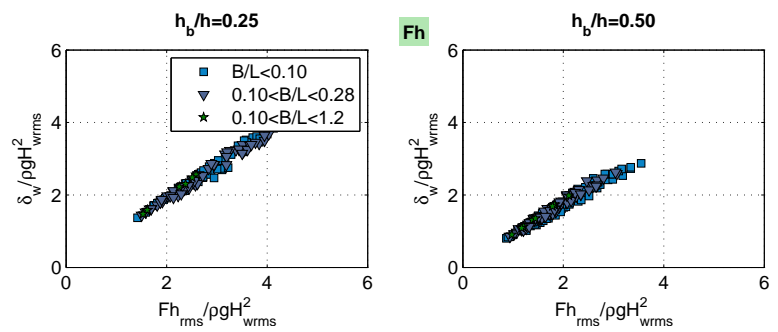

Figure 17: Non-dimensional value of $\delta_{w}$ compared with the non-dimensional value the r.m.s of $F h$ and $F v$ as a function of $B / L$ and $h_{b} / h$. Type $B$.
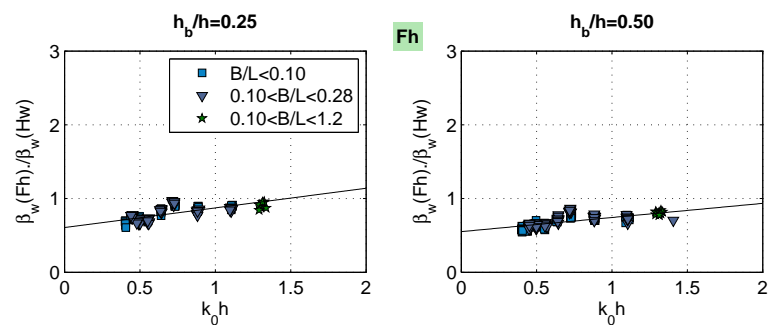

Figure 18: Relationship between $\beta_{w}$ in Weibull pfd of $F h$ or $F v$ and $H_{w}$ as a function of $k_{0} h, B / L$ and $h_{b} / h$. Type B.

Joint cumulative distribution of horizontal and vertical forces

If $V_{1}$ and $V_{2}$ are two variables with a Weibull pdf described by $\delta_{w 1}, \beta_{w 1}$ and $\delta_{w 2}, \beta_{w 2}$, the scale and shape parameters respectively; and $0 \leq \rho_{r} \leq 1$ is the linear correlation coefficient defined as:

$$
\rho_{r}=\frac{\operatorname{Cov}\left(V_{1}, V_{2}\right)}{\sqrt{\sigma_{s}^{2}\left(V_{1}\right) \sigma_{s}^{2}\left(V_{2}\right)}}
$$

the joint probability density function of the normalized variables $V_{1} / \delta_{w 1}$ and $V_{2} / \delta_{w 2}$ can be defined as (Yacoub et al., 2005):

$$
p\left(v_{1}, v_{2}\right)=\frac{\beta_{w 1} \beta_{w 2} v_{1}^{\beta_{w 1}-1} v_{2}^{\beta_{w 2}-1}}{1-\rho_{r}} \exp \left(-\frac{v_{1}^{\beta_{w 1}}+v_{2}^{\beta_{w 2}}}{1-\rho_{r}}\right) I_{0}\left(\frac{2 \sqrt{\rho_{r} v_{1}^{\beta_{w 1}} v_{2}^{\beta_{w 2}}}}{1-\rho_{r}}\right)
$$




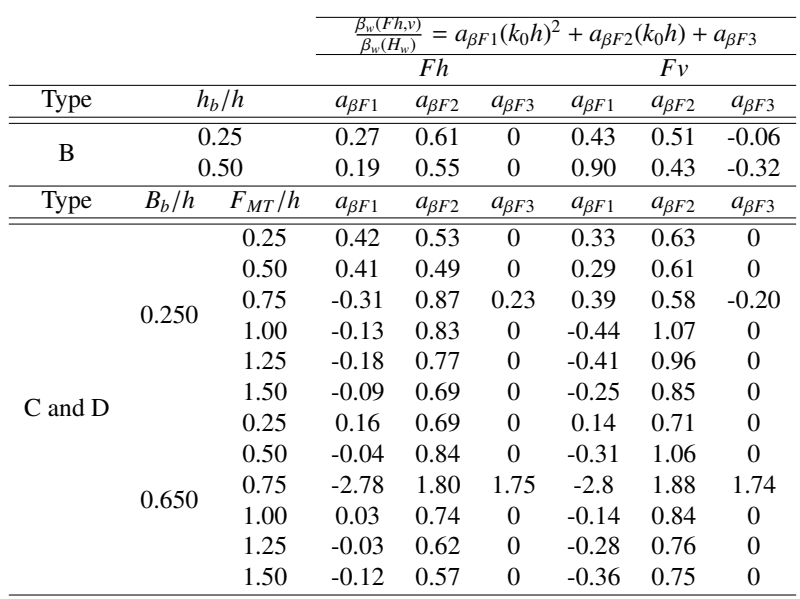

Table 4: Best fit to calculate the relationship between $\beta_{w}$ in Weibull pfd of $F h$ or $F v$ and $H_{w}$.

where $I_{0}$ is the modified Bessel function of the first kind and order 0 (Abramowitz and Stegun, 1972). Joint cumulative distribution can be obtain from 15 as:

$$
P\left(v_{1}, v_{2}\right)=\iint p\left(v_{1}, v_{2}\right) d v_{1} d v_{2}
$$

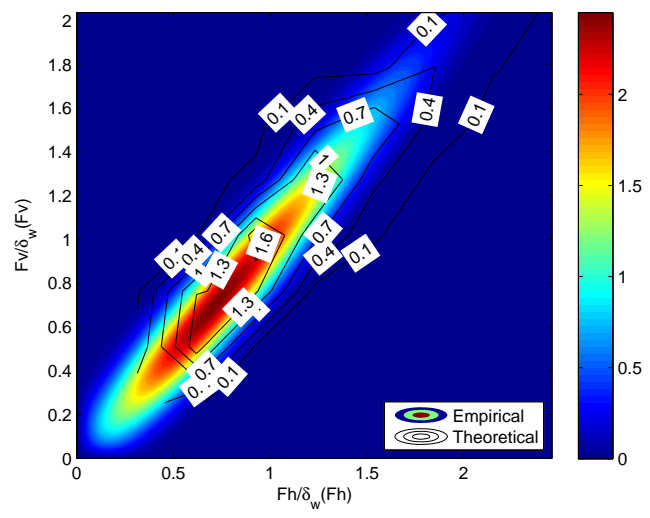

Figure 19: Joint probability function of $F h$ and $F v$. Type $\mathbf{C}, F_{M T} / h=\mathbf{0 . 7 5}, B_{b} / h=\mathbf{0 . 2 5}, T_{p t e o}=\mathbf{1 . 0 5} \mathbf{s}$, $H_{I s ~ t e o}=\mathbf{0 . 0 4} \mathbf{m}$ and $D=40 \mathrm{~mm}$. Empirical and theoretical fit (equation 15).

Joint pdf of the normalized $F h$ and $F v\left(F h / \delta_{w}(F h)\right.$ and $F v / \delta_{w}(F v)$ respectively) has been obtained. Figure 19 shows the empirical joint pdf juxtaposed with the theoretical one calculated with equation 15 for a breakwater type B with $F_{M T} / h=0.75, B_{b} / h=0.25, T_{p \text { teo }}=1.05 \mathrm{~s}, H_{I s \text { teo }}=0.04 \mathrm{~m}$ and $D=40 \mathrm{~mm}$. Results show that the joint pdf of $F h / \delta_{w}(F h)$ and $F v / \delta_{w}(F v)$ fits fairly good to the theoretical pdf defined with equation 15. The value of $\rho_{r}$ is presented in figure 20 for Type B (as example) with the fit to calculate it given in table 5 as a function of the breakwater typology. Results show that $\rho_{r}$ decreases whit the water depth increases for all breakwater typologies. Moreover, its value depends on the other parameters defining the breakwater typology.

\section{Calculation scheme}

For a given sea state $\left[H_{\text {Irms }}, T_{p}\right]$, r.m.s incident wave height and peak period, with normal incidence, and a pre-design breakwater typology, the methodology to calculate the failure probability of the breakwater for sliding associated with this sea state is the following:

1 To calculate $K_{R}$ and $\phi$ from the hydraulic performance curves (Vílchez et al., 2016). 


\begin{tabular}{|c|c|c|c|c|}
\hline & & & & $\rho_{r}=a_{\rho_{r}} e^{k_{0} h}+1$ \\
\hline Type & $h_{b} / h$ & \multicolumn{2}{|l|}{$B / L$} & $a_{\rho_{r}}$ \\
\hline \multirow{6}{*}{ B } & \multirow{3}{*}{0.25} & \multicolumn{2}{|l|}{$B / L<0.10$} & -0.012 \\
\hline & & \multicolumn{2}{|c|}{$0.10 \leq B / L<0.28$} & -0.010 \\
\hline & & \multicolumn{2}{|c|}{$0.28 \leq B / L<1.20$} & -0.025 \\
\hline & \multirow{3}{*}{0.50} & \multicolumn{2}{|l|}{$B / L<0.10$} & -0.022 \\
\hline & & \multicolumn{2}{|c|}{$0.10 \leq B / L<0.28$} & -0.026 \\
\hline & & \multicolumn{2}{|c|}{$0.28 \leq B / L<1.20$} & -0.025 \\
\hline Type & $B_{b} / h$ & $B / L$ & $F_{M T} / h$ & $a_{\rho_{r}}$ \\
\hline \multirow{12}{*}{ C and D } & \multirow{6}{*}{0.250} & \multirow{6}{*}{$0.10<B / L<0.35$} & 0.25 & -0.012 \\
\hline & & & 0.50 & -0.011 \\
\hline & & & 0.75 & -0.021 \\
\hline & & & 1.00 & -0.021 \\
\hline & & & 1.25 & -0.013 \\
\hline & & & 1.50 & -0.010 \\
\hline & \multirow{6}{*}{0.625} & \multirow{6}{*}{$0.10<B / L<0.35$} & 0.25 & -0.010 \\
\hline & & & 0.50 & -0.010 \\
\hline & & & 0.75 & -0.010 \\
\hline & & & 1.00 & -0.021 \\
\hline & & & 1.25 & -0.020 \\
\hline & & & 1.50 & -0.047 \\
\hline
\end{tabular}

Table 5: Best fit to calculate the linear correlation coefficient between $F h$ and $F v$ as a function of $k_{0} h$ and $B / L$.
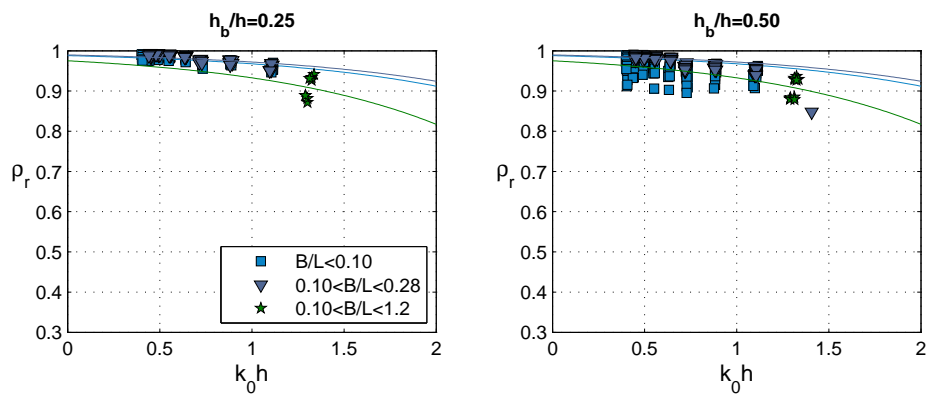

Figure 20: Linear correlation coefficient between $F h$ and $F v$ as a function of $k_{0} h$ and $B / L$. Type B.

2 To obtain the r.m.s value of the total wave height at the breakwater toe, $H_{p r m s}$ as:

$$
H_{p r m s}(x)=H_{\text {Irms }} \sqrt{1+K_{R}^{2}+2 K_{R} \cos \left(2 k_{0} x+\phi\right)}
$$

3 To calculate the r.m.s value of the total wave height at the breakwater wall, $H_{w r m s}$. This can be obtained following Vílchez et al. (2017) from the Weibull pdf as a function of $H_{p r m s}, \alpha_{w}$ and $\beta_{w}$ as:

$$
H_{w r m s}=\sqrt{\left(\alpha_{w} H_{p r m s}\right)^{2} \Gamma\left(1+\frac{2}{\beta_{w}}\right)-\left[\alpha_{w} H_{p r m s} \Gamma\left(1+\frac{1}{\beta_{w}}\right)\right]^{2}}
$$

where $\Gamma$ is the gamma function.

4 To obtain r.m.s value of the horizontal force by integrating the pressure law at the breakwater wall (figure 21). The final expression as a function of $H_{w r m s}$ is given by:

- For type $\mathrm{C}$ and $F_{M T} / h=0.75$ (Figure $21 \mathrm{~b}$ ):

$$
F h_{r m s}=\rho g \frac{H_{w r m s}}{8}\left\{H_{w r m s}+2\left(h-h_{b}\right)\left(1+\frac{\cosh \left(k_{0} h_{b}\right)}{\cosh \left(k_{0} h\right)} \alpha_{2}\right\} \alpha_{1} \alpha_{I}\right.
$$

- For the rest of the typologies (Figure $21 \mathrm{a}$ ): 


$$
F h_{r m s}=\rho g \frac{H_{w r m s}}{8}\left\{H_{w r m s}+2\left[\left(h-F_{M T}\right)+\left(h-h_{b}\right) \frac{\cosh \left(k_{0} F_{M T}\right)}{\cosh \left(k_{0} h\right)}+\left(F_{M T}-h_{b}\right) \frac{\cosh \left(k_{0} h_{b}\right)}{\cosh \left(k_{0} h\right)} \alpha_{2}\right]\right\} \alpha_{1} \alpha_{I}
$$

where $\alpha_{1}=1.14 ; \alpha_{I}$ is given in figure 6 for HMCB and $\alpha_{I}=1$ for the rest of typologies; and $\alpha_{2}$ is given in table 1 .

5 To calculate $F v_{r m s}$ with $\alpha_{4}$ (table 3 ) as follows:

$$
F v_{r m s}=F h_{r m s} \alpha_{4}
$$

6 To evaluate the bi-parametric Weibul pdf of $F h$ and $F v$. The scale parameters are $\delta_{w}=F h_{r m s}$ or $F v_{r m s}$ respectively. The shape parameter, $\beta_{w}$ can be calculated as a function of $\beta_{w}\left(H_{w}\right)$ as given in table 4.

7 To obtain the joint pdf of $[F h, F v]$ with equation 15. The value of $\rho_{r}$ can be calculated as given in table 5.

8 Applying simulation technique series of $F h$ and $F v$ can be obtained from its marginal and joint pdfs, and the pdf of the safety margin $S$ can be obtained for this sea state.

The failure probability of the breakwater in its useful life for this failure mode can be estimated by repeating the previous process for the different sea states (with an associated probability of occurrence) that can occur in the useful life of the breakwater.
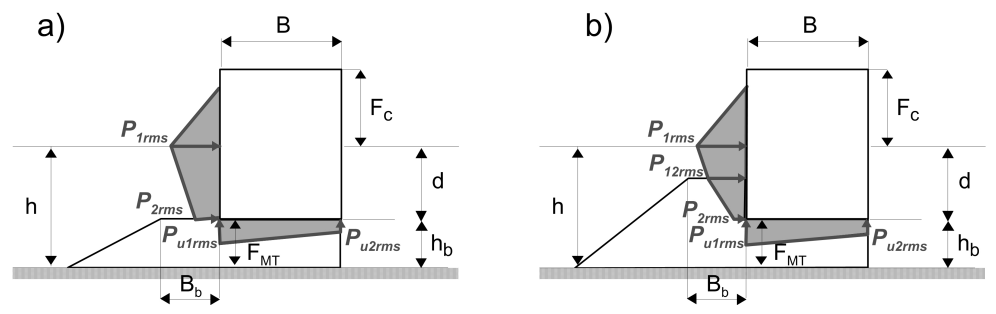

Figure 21: Scheme of pressure.

\section{CONCLUSIONS}

An unified method has been proposed to simultaneously verify the failure modes produced by the same wave conditions which affect the front or face of the breakwater. The methodology is based on the derivation of the pdf of the kinematic and dynamic variables of the total oscillation in front of the breakwater as a function of the total wave height at that same location. These variables define the actions in the breakwater and are necessary to formulate the verification equation of the failure modes. This methodology has been developed for the failure mode that considers sliding of the superstructure. The following conclusions have been obtained:

1 The r.m.s of the dynamic pressure in the wave crest at the breakwater wall and at the bottom of the caisson is directly proportional to the r.m.s wave height at this location. An approximation to calculate the r.m.s of these pressure laws has been proposed based on the evaluation of the transfer function between these variables for a representative frequency.

2 The relation between the non-dimensional values of the horizontal and vertical forces in the crown wall is linear. Three force regimes have been defined as a function of $B / L$ depending on which force predominates.

3 The probability density functions of the horizontal and vertical forces in the wave crest follow a biparametric Weibull distribution. The scale parameter is the r.m.s of these variables, which can be obtained directly from the total wave height at the wall applying the developed pressure scheme. 
4 It is noted that the maximum horizontal and uplift force are not always in phase. The joint probability density function of the horizontal and vertical forces in wave crest can be calculated with parameters related to those of the marginal Weibull distribution of these variables and the linear correlation coefficient which depend on the relative depth and the breakwater typology.

5 The safety margin in the verification equation can be obtained from the horizontal and vertical forces in the wave crest of a given sea state. The failure probability of the breakwater in its useful life for this failure mode can be estimated by applying the previous process for the different sea states (with an associated probability of occurrence) that can occur in the useful life of a breakwater. The verification equation of the other failure modes produced by the same agent, wind waves, in the front or face of the breakwater could be obtained in the same way, and to simultaneously verify all of these failure modes.

\section{References}

A. Abramowitz and I. A. Stegun. Handbook of Mathematical Functions. New York, Dover, 1972.

A. Baquerizo. Reflexión del oleaje en playas. Métodos de evaluación y de predicción. $\mathrm{PhD}$ thesis, Cantabria, 1995.

L. Borgman. Random Hydrodynamic Forces on Objects. Ann. Math Statist., 38:37-51, 1967.

M. Clavero, M. Vílchez, D. Pérez., M. I. Benedicto, and M. A. Losada. An unified desing method of maritime works against waves. In Coastal Engineering Proceedings, 1(33), structures.76, pages 1-9, 2012.

Y. Goda. Random seas and desing of maritime structures. University of Tokio Press, 1985.

J. L. Lara, I. J. Losada, and R. Guanche. Wave interaction with low-mound breakwaters using a RANS model. Ocean Engineering, 35(13):1388-1400, sep 2008.

I. J. Losada, R. A. Dalrymple, and M. A. Losada. Water waves on crown Breakwaters. Journal of Waterway, Port, Coastal and Ocean Engineering, 119(4):367-380, 1993.

ROM 1.0. ROM 1.0. Recomendaciones geotécnicas para obras marítimas y portuarias. Puertos del Estado, 2009. ISBN 9788488975737. URL http://www .puertos .es/programa_rom/rom_10_09_en. html.

S. Takahashi. Design of vertical breakwaters. Port and Harbor Research Institute, Reference Document, Japan., 34, 1996.

M. Vílchez, J. Moyano, M. Clavero, M. D. Miguel, and M. A. Losada. Forces and subpressures on nonovertoppable vertical breakwaters. In Proceedings of the 6th International Conference of Coastal Structures, pages 545-555, 2011.

M. Vílchez, M. Clavero, and M. A. Losada. Hydraulic performance of different non-overtopped breakwater types under 2D wave attack. Coastal Engineering, 107:34-52, 2016.

M. Vílchez, M. Clavero, J. L. Lara, and M. A. Losada. A characteristic friction diagram for the numerical quantifcation of the hydraulic performance of different breakwater types. Coastal Engineering, 114: 86-98, 2016b.

M. Vílchez, M. Clavero, A. Baquerizo, and M. A. Losada. An approximation to the statistical characteristics of wind waves in front and from the toe of the structure to the toe of the crown of non-overtopped breakwaters. Under review in Coastal Engineering Journal, 2017.

P. WG47. Criteria for the selection of breakwater types and their related optimum safety levels. PIANC, 2013.

M. D. Yacoub, D. Benevides, U. S. Dias, and G. Fraidenraich. Joint Statistics for Two Correlated Weibull Variates. IEEE Antennas and Wireless Propagation Letters, 4(February):129-132, 2005. ISSN 15361225. doi: 10.1109/LAWP.2005.845910. 